\title{
Measurement of the zero-field magnetic dipole moment of magnetizable colloidal silica spheres
}

\author{
E M Claesson, B H Erné, I A Bakelaar, B W M Kuipers and A P Philipse \\ Van 't Hoff Laboratory for Physical and Colloid Chemistry, Debye Institute, Utrecht University, \\ Padualaan 8, $3584 \mathrm{CH}$ Utrecht, The Netherlands \\ E-mail: e.m.claesson@chem.uu.nl
}

Received 14 November 2006, in final form 8 December 2006

Published 5 January 2007

Online at stacks.iop.org/JPhysCM/19/036105

\begin{abstract}
The magnetic properties of dispersions of magnetic silica microspheres have been investigated by measuring the magnetization curves and the complex magnetic susceptibility as a function of frequency and field amplitude. The silica spheres appear to have a net permanent magnetic dipole moment, even in zero field, which is increased significantly after a temporary exposure of the silica colloids to a saturating magnetic field. The magnetic properties of the microparticles in zero field are discussed in terms of the number and the orientations of the embedded nanoparticle dipoles along an easy axis of magnetization in the absence of an external field.
\end{abstract}

(Some figures in this article are in colour only in the electronic version)

\section{Introduction}

Magnetic microspheres used in biomedical applications generally do not have a permanent magnetic moment [1]. The particles become magnetized in an external magnetic field and lose their magnetization when the field is removed. In our laboratory, silica microspheres have been developed with embedded cobalt ferrite nanoparticles [2]. At room temperature a large fraction of the magnetic moments of the nanoparticles is blocked and, as a result, the microspheres have a permanent magnetic moment. Such particles can be used in fundamental studies of the rotational diffusion of colloidal spheres using complex magnetic susceptibility measurements, which are being carried out at fields which in strength are comparable to the earth magnetic field, that is, too weak to exert a significant torque on the particles. The interaction of the particles with the field is comparable to the thermal energy, so the reorientation of the particles with the applied magnetic field is diffusive. Using magnetic methods has several advantages compared to previously used methods such as depolarized dynamic light scattering (DDLS) [3], time-resolved phosphorescence anisotropy (TPA) [4], or polarized fluorescence recovery after photobleaching (pFRAP) [5]. The magnetic measurements are not restricted to low particle 
concentrations or solvents with a specific index of refraction since the particles do not have to be optically matched with the surrounding medium. Moreover, problems such as luminescencereabsorption effects are avoided [4,5], and at a particular concentration, it is possible to obtain the distribution of diffusion coefficients directly rather than just one average value.

For a proper interpretation of rotational diffusion experiments using a magnetic technique, it is important that the magnetic properties, such as the magnetic dipole moment, of the colloidal dispersion, can be quantified. The aim of the present work is to provide this quantification for cobalt ferrite-doped silica microparticles by analysing three types of magnetic measurements: magnetization curves, complex magnetic susceptibility spectra in the low-field limit, and the field-amplitude dependence of the complex magnetic susceptibility in the low-frequency limit. The magnetic dipole moment of microparticles in zero field is determined before and after exposure to a strong magnetic field to investigate whether the dipole moment of the colloids can be permanently increased.

The outline of this paper is as follows. In section 2, the theoretical background of magnetization measurements and complex magnetic susceptibility spectroscopy is reviewed. In section 3, the technical equipment, preparation of the magnetic particles and the experimental procedures are described, and in section 4, the results from the measurements are presented. In section 5, the magnetic properties of the particles and the dispersions will be discussed.

\section{Theory}

\subsection{Magnetization curves}

The magnetization $M$ measured as a function of the applied magnetic field $H$ is investigated for (a) dispersions and dry powder of cobalt ferrite nanoparticles and (b) dispersions and dry powder of the silica microspheres with embedded cobalt ferrite. The equations involved in the analysis of the magnetization curves are presented below.

The distribution of magnetic dipole moments of the nanoparticles can be determined from the magnetization curve of dilute nanoparticle dispersions, for which dipole-dipole interactions have a negligible effect. In line with observations using electron microscopy, it is assumed that the magnetic diameter, $d_{\mathrm{m}}$, is lognormally distributed:

$$
P\left(d_{\mathrm{m}}\right)=\frac{1}{\sigma d_{\mathrm{m}} \sqrt{2 \pi}} \exp \left[\frac{-\ln ^{2}\left(d_{\mathrm{m}} / d_{0}\right)}{2 \sigma^{2}}\right] .
$$

Here, $P\left(d_{\mathrm{m}}\right)$ is the probability density function, $\sigma^{2}$ is the variance, and $d_{0}$ is the magnetic diameter at the maximum of the distribution. The dipole moment, $\mu_{\mathrm{np}}$, of a single magnetic nanoparticle is given by

$$
\mu_{\mathrm{np}}=\frac{\pi}{6} d_{\mathrm{m}}^{3} m_{\mathrm{s}}
$$

where $m_{\mathrm{s}}$ is the material-dependent saturation magnetization per unit volume $\left(425 \mathrm{kA} \mathrm{m}^{-1}\right.$ for cobalt ferrite [6]). It is assumed that all nanoparticles have a single magnetic domain. The shape of the magnetization curve of monodisperse nanoparticles in dispersion is determined by the Langevin equation:

$$
M=M_{\mathrm{s}} L(\alpha)=M_{\mathrm{s}}\left[\operatorname{coth}(\alpha)-\frac{1}{\alpha}\right]
$$

where $M_{\mathrm{s}}$ is the saturation magnetization and

$$
\alpha=\frac{\mu_{0} \mu H}{k_{\mathrm{B}} T} .
$$


Here, $k_{\mathrm{B}} T$ is the thermal energy and $\mu_{0}=4 \pi \times 10^{-7} \mathrm{~J} \mathrm{~A}^{-2} \mathrm{~m}^{-1}$ the magnetic permeability of vacuum. For nanoparticles with a lognormal distribution at low concentrations where interactions are negligible, the magnetization of the dispersion at field $H$ is given by

$$
M=M_{\mathrm{s}} \frac{\int_{0}^{\infty} P(\mu) \mu L(\alpha(\mu, H)) \mathrm{d} \mu}{\int_{0}^{\infty} P(\mu) \mu \mathrm{d} \mu}
$$

with the function $L(\alpha)$ defined via (3); $P(\mu)$ is the probability density function for the particle dipole moment, $\mu$. The mean magnetic diameter and the width of its distribution are obtained from a theoretical fit of equation (5) to the magnetization curve of the nanoparticle dispersion. The permanent magnetic moment of microparticles cannot be obtained in the same way, since they also have an induced dipole moment. Alternative approaches are discussed in the next two sections.

If individual magnetic moments are blocked inside the cobalt ferrite nanoparticles, magnetization curves of dry nano- or microparticles will display hysteresis. The timescale for the rotation of the magnetic moment inside the nanoparticles, so-called Néel relaxation, strongly depends on the magnetic particle volume, $V_{\mathrm{m}}[7]$ :

$$
\tau_{\mathrm{N}}=\frac{\sqrt{\pi}}{2} \tau_{0} \cdot \exp \left[\frac{K V_{\mathrm{m}}}{k_{\mathrm{B}} T}\right]
$$

where $\tau_{0}=10^{-9} \mathrm{~s}$ and $K$ is the anisotropy constant (200 $\mathrm{kJ} \mathrm{m}^{-3}$ for cobalt ferrite [7]). According to (6), cobalt ferrite nanoparticles with a diameter of $10 \mathrm{~nm}$ or more have a blocked orientation of the magnetic dipole moment at room temperature $\left(\tau_{\mathrm{N}}>100 \mathrm{~s}\right)$.

\subsection{Complex magnetic susceptibility spectra in the low-field limit}

The permanent magnetic moment as well as the hydrodynamic radius of magnetic microparticles can be determined by measuring the frequency-dependent complex magnetic susceptibility of a dispersion of the particles [8]. If the particles have a permanent magnetic moment, the real part of the magnetic susceptibility will have a plateau value in the lowfrequency limit and will decrease with increasing frequency when approaching the frequency of Brownian rotation of the microparticles (see figure 1) to finally decrease to zero in the high-frequency limit. At the characteristic frequency, the imaginary part of the susceptibility spectrum will have a maximum. With this technique, the permanent magnetic moment of the microparticles is separated from other contributions to the susceptibility, such as the fieldinduced alignment of nanoparticle dipoles that are not blocked or of free nanoparticles, which are not embedded in microparticles. Néel and Brownian relaxation of single nanoparticles occur at much higher frequencies than the frequency of Brownian rotation of the microspheres.

In the limit of low field and low frequency, the dimensionless magnetic susceptibility $\chi=M / H$ of a colloidal dispersion consists of two contributions:

$$
\chi=\chi_{\mathrm{LF}}+\chi_{\mathrm{HF}}
$$

The contribution $\chi_{\mathrm{LF}}$ is present only at frequencies low enough for the orientation of the permanent dipole moment of microspheres to respond to the weak alternating external magnetic field by Brownian rotation:

$$
\chi_{\mathrm{LF}}=\frac{N_{\mathrm{mp}}}{V} \frac{\mu_{0}\left(\mu_{\mathrm{mp}}\right)^{2}}{3 k_{\mathrm{B}} T} .
$$

Here, $N_{\mathrm{mp}}$ is the number of composite microparticles, $V$ is the sample volume, and $\mu_{\mathrm{mp}}$ is the permanent magnetic moment of the microparticles. Equation (8) is obtained by performing a Taylor expansion of (3). From (8), measurement of the initial susceptibility of a sample with 


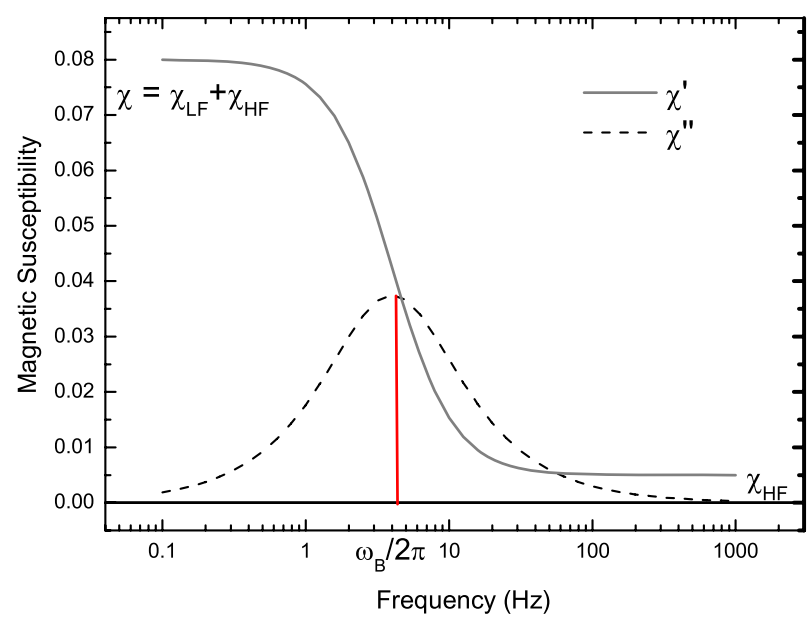

Figure 1. Calculated complex magnetic susceptibility spectrum for microspheres with a permanent magnetic dipole moment and a hydrodynamic radius, $R_{\mathrm{h}} \sim 200 \mathrm{~nm}$ in ethanol (solvent viscosity $\eta=1.074 \mathrm{mPa}$ s at $T=298.15 \mathrm{~K} \mathrm{[9]).} \mathrm{At} \mathrm{the} \mathrm{characteristic} \mathrm{frequency} \mathrm{for} \mathrm{Brownian} \mathrm{rotation}$ of these particles, $4 \mathrm{~Hz}$, the real component $\chi^{\prime}$ has the highest frequency dependence and the imaginary component $\chi^{\prime \prime}$ has a maximum $(10 a)-(10 c)$. The dipole moment of the microspheres can be obtained from the susceptibility in the low-frequency limit, $\chi_{\mathrm{LF}}$, minus the susceptibility in the high-frequency limit, $\chi_{\mathrm{HF}}$. The latter is proportional to the number of nanoparticles with non-blocked dipoles.

a known concentration $N_{\mathrm{mp}} / V$ allows calculation of $\mu_{\mathrm{mp}}$. The second contribution, $\chi_{\mathrm{HF}}$, is present at higher frequencies too where the contribution $\chi_{\mathrm{LF}}$ of the microspheres has been lost. This is due to nanoparticles that respond individually, by Néel relaxation or Brownian rotation, $\omega_{\mathrm{B}}$, in the solvent. The characteristic frequency determined by Brownian rotation of particles in a solvent is given by $[10,11]$

$$
\omega_{\mathrm{B}}=2 D_{\mathrm{r}}=\frac{k_{\mathrm{B}} T}{4 \pi \eta R_{\mathrm{h}}^{3}}
$$

where $D_{\mathrm{r}}$ is the rotational diffusion coefficient, $\eta$ is the viscosity of the solvent, and $R_{\mathrm{h}}$ is the hydrodynamic radius of the spherical particles. At this frequency, the in-phase, real component $\chi^{\prime}$ of the complex susceptibility has the highest frequency dependence and the out-of-phase, imaginary component $\chi^{\prime \prime}$ has an extremum $[10,11]$ :

$$
\begin{aligned}
& \chi(\omega)=\chi^{\prime}(\omega)-\mathrm{i} \chi^{\prime \prime}(\omega) \\
& \chi^{\prime}(\omega)=\chi_{0} \frac{\omega_{\mathrm{B}}^{2}}{\omega_{\mathrm{B}}^{2}+\omega^{2}} \\
& \chi^{\prime \prime}(\omega)=\chi_{0} \frac{\omega_{\mathrm{B}} \omega}{\omega_{\mathrm{B}}^{2}+\omega^{2}}
\end{aligned}
$$

Here, $\omega=2 \pi f$ is the angular frequency in $\mathrm{s}^{-1}$ and $f$ is the cycle frequency in Hz. From (9), for a solvent viscosity of $\eta \approx 1 \mathrm{mPa}$ s at $T=298 \mathrm{~K}$, the characteristic frequencies for the Brownian rotation of composite particles with a radius of $100-1000 \mathrm{~nm}$ is in the range $0.05-50 \mathrm{~Hz}$, whereas that of nanoparticles of $1-10 \mathrm{~nm}$ in radius is in the $0.05-50 \mathrm{MHz}$ range, 3-6 orders of magnitude higher. The contributions of microparticles and single nanoparticles dipoles can therefore clearly be separated in a complex magnetic susceptibility spectrum. 
Table 1. Characterization parameters of the magnetic silica dispersions.

\begin{tabular}{llll}
\hline & Sample 1 & Sample 2 & Sample 3 \\
\hline Nanoparticles & Cobalt ferrite & Cobalt ferrite & Maghemite \\
$R_{\text {TEM }}(\mathrm{nm})^{\mathrm{a}}$ & $187 \pm 34$ & $409 \pm 9$ & $154 \pm 11$ \\
$\rho_{\mathrm{p}}\left(\mathrm{g} \mathrm{ml}^{-1}\right)^{\mathrm{b}}$ & 2.45 & 2.14 & - \\
$N_{\mathrm{mp}} / V\left(\mathrm{~m}^{-3}\right)^{\mathrm{c}}$ & $3.53 \times 10^{17}$ & $1.32 \times 10^{17}$ & - \\
\hline
\end{tabular}

${ }^{a}$ Particle radius obtained from analysis of TEM images.

b Particle mass density.

c Particle number concentration.

\subsection{Field-amplitude dependence of the low-frequency magnetic susceptibility}

A second approach to determine the permanent magnetic dipole moment of microparticles is from the field-amplitude dependence of the low-frequency magnetic susceptibility. The dimensionless magnetic susceptibility $\chi$ is given by

$$
\chi=\frac{M}{H} \sim \frac{M_{\mathrm{s}} L(\alpha)}{H} .
$$

In the low-field limit the Langevin equation (3) is linear:

$$
L(\alpha)_{H \rightarrow 0}=\frac{\alpha}{3}
$$

and the initial magnetic susceptibility, $\chi_{0}$, is independent of $H$. Beyond the low-field limit, $\chi$ starts to change. The deviation from $\chi_{0}$ can be expressed by taking the ratio of $M / H$ with the low-field limit value. Assuming that the field is still too low to change the magnetic moment of the microspheres or to induce dipolar structures, the following expression is obtained:

$$
\frac{\chi}{\chi_{0}}=\frac{L(\alpha)}{\alpha / 3}=\frac{3}{\alpha}\left[\operatorname{coth}(\alpha)-\frac{1}{\alpha}\right] \text {. }
$$

When the microspheres have a large permanent magnetic moment, the Langevin equation deviates from the linear approximation in (12) at fields well below $1 \mathrm{kA} \mathrm{m}^{-1}$. The magnetic moment can be calculated from this deviation from linear behaviour by fitting the expression in (13) to a plot of $\chi / \chi_{0}$ versus the field amplitude $H$ measured at constant temperature. From the obtained value for $\alpha$ (4) the dipole moment $\mu_{\mathrm{mp}}$ of the microspheres can be calculated without knowing the concentration of particles in the measured dispersion.

\section{Experimental details}

Magnetic silica colloids were prepared by adding an aqueous ferrofluid of cobalt ferrite [12] or maghemite [13] nanoparticles to thiol-functionalized colloidal silica spheres [2]. The resulting core-shell microspheres were coated with an outer silica shell, and the surface was grafted with 3-(trimethoxysilyl)propyl methacrylate (TPM) to stabilize the particles [14]. The particle dispersions were purified extensively by sedimentation and redispersion in ethanol. A more detailed description of the synthesis of magnetic silica particles can be found in [2]. Three dispersions of magnetic microspheres were studied in this work (see table 1): two silicacobalt ferrite dispersions with different microparticle sizes - where a large fraction of the dipole moments of the nanoparticles is assumed to be blocked-as well as one dispersion of silicamaghemite particles.

The particles were characterized with transmission and scanning electron microscopy (TEM and SEM). The complex magnetic susceptibility of dispersions 1 and 2 was measured 
before and after the particles had been exposed to a homogeneous field of $2 \mathrm{~T}$ for $1 \mathrm{~h}$, using a Bruker BE 25v electromagnet. Magnetization curves of the samples and the corresponding nanoparticles in dispersion as well as in powder form were measured with a Micromag 2900 alternating gradient magnetometer (Princeton Measurements Corp.). The dispersion samples were contained in rectangular glass sample holders of $4 \mathrm{~mm} \times 4 \mathrm{~mm} \times 0.4 \mathrm{~mm}$ with the square sides parallel to the magnetic field direction to minimize demagnetization. The dry powders were fixed to a thin glass slide of dimensions $7 \mathrm{~mm} \times 3 \mathrm{~mm}$ with adhesive tape.

Complex magnetic susceptibility measurements were performed using a setup described in detail elsewhere [15]. It is a new version of an earlier setup [10] now with higher sensitivity and possibility to measure at lower frequencies. In brief, the setup is a differential transformer with two multilayered cylindrical copper coils: a primary layer through which an applied alternating current flows and a secondary layer in which an alternating voltage is induced. The key idea of the setup is to use secondary coils with a maximized number of layers beyond which the signal would no longer increase but drop due to capacitance and absorption effects. An AC current is applied to the primary coils using a Yokogawa FG120 function generator and the voltage induced in the secondary coils is measured using a 7265 Signal recovery differential lock-in amplifier in the $2 \mathrm{mHz}$ to $1 \mathrm{kHz}$ range. The colloidal dispersion of magnetic microparticles is contained in a cylindrical glass tube. When the magnetic sample is introduced in the coils, the change in the measured alternating voltage is proportional to the complex susceptibility of the sample. The amplitude of the alternating magnetic field used for the experiments presented here was relatively low, $57 \mathrm{~A} \mathrm{~m}^{-1}$ if not specified otherwise, to avoid changing the magnetic structure of the samples. The sample and coils were kept at a constant temperature of $295.5 \mathrm{~K}$ in a thermostatized and grounded box made of mu-metal for shielding from external lowfrequency magnetic interference.

To calculate the magnetic dipole moment of the microspheres according to (8), the particle number concentration $\left(N_{\mathrm{mp}} / V\right)$ must be determined. A small amount of the measured dispersions 1 and 2 with known weight, $m_{\text {disp }}$, and volume, $V_{\text {disp }}$, was dried to obtain the weight of dry particles, $m_{\mathrm{p}}$. $V_{\text {disp }}$ was obtained by measuring the height of the dispersion with a cathetometer inside a calibrated tube with a known volume-height relation. Together with the solvent density, $\rho_{\mathrm{s}}=0.789 \mathrm{~g} \mathrm{ml}^{-1}$, the mass density of the particles, $\rho_{\mathrm{p}}$ was calculated:

$$
\rho_{\mathrm{p}}=\frac{m_{\mathrm{p}}}{V_{\text {disp }}-\left(\frac{m_{\text {disp }}-m_{\mathrm{p}}}{\rho_{\mathrm{s}}}\right)} .
$$

The sample volume, $V_{\text {sample }}$, of the measured dispersion of sample 1 and 2 was obtained in the same way as described above. The number concentration of particles was obtained from

$$
\frac{N_{\mathrm{mp}}}{V}=\frac{3 \phi_{\mathrm{m}} m_{\mathrm{disp}}}{4 \pi\left(R_{\mathrm{TEM}}\right)^{3} \rho_{\mathrm{p}} V_{\text {sample }}},
$$

where $\phi_{\mathrm{m}}=m_{\mathrm{p}} / m_{\mathrm{disp}}$ is the mass fraction of particles and $R_{\mathrm{TEM}}$ is the physical radius of the particles.

\section{Results}

\subsection{Magnetization curves}

The magnetic moment of the cobalt ferrite nanoparticles can be determined from the magnetization curve of the particles in a dilute dispersion (figure 3(a)). A fit of the data based on (5) gives a magnetic moment of $1.61 \times 10^{-19} \mathrm{~A} \mathrm{~m}^{2}$ with a diameter distribution width given by $\sigma=0.51$ (1). A magnetization curve of the same nanoparticles but dry (figure 3(b)) 
clearly shows hysteresis, with a remanence $M_{\mathrm{r}} / M_{\mathrm{s}}$ of 0.425 . For the nanoparticles, a saturation magnetization of $m_{\mathrm{s}}=240 \mathrm{kA} \mathrm{m}^{-1}$ is found $\left(m=0.27 \mathrm{mg}, \rho_{\mathrm{p}}=4907 \mathrm{~kg} \mathrm{~m}^{-3}\right.$ [7], $M_{\mathrm{s}}=1.32 \times 10^{-19} \mathrm{~A} \mathrm{~m}^{2}$ ). This is much lower than the $m_{\mathrm{s}}=425 \mathrm{kA} \mathrm{m}^{-1}$ for cobalt ferrite as reported in the literature [7]. The reason for this is that such nanoparticles have a non-magnetic iron oxide shell [12], which still has roughly the same mass density as cobalt ferrite [7,9]. With an average magnetic diameter of about $11 \mathrm{~nm}$, a large fraction of the cobalt ferrite particles has a radius large enough for the dipole moments to be blocked at room temperature.

A practical difficulty when measuring a magnetization curve of the dispersed magnetic silica particles (figure 3(c)) is their sedimentation rate. This is already high in zero field $\left(0.22 \mathrm{~mm} \mathrm{~h}^{-1}\right)$ and is much enhanced in an external magnetic field. Large magnetic aggregates are formed that sediment rapidly while the magnetization curve is being measured. These large structures and the sediment lead to hysteresis, since Brownian relaxation is slowed down significantly. It was also observed that the remanence in the sample 1 powder, $M_{\mathrm{r}} / M_{\mathrm{s}}=0.305$, is lower than for the pure cobalt ferrite particles in the dry state. However, from the saturation magnetization of dry microparticles $\left(2.06 \times 10^{-3} \mathrm{~A} \mathrm{~m}^{2} \mathrm{~g}^{-1}\right.$, figure $\left.3(\mathrm{~d})\right)$, valuable information can still be obtained. The total amount of magnetic material can be calculated based on the weight of microparticles, which for sample 1 (see table 1) corresponds to $42.1 \mathrm{mg}$ nanoparticles per gram of microspheres.

\subsection{Frequency-dependent complex magnetic susceptibility}

In figure 4, a spectrum is shown of silica microparticles doped with maghemite. In that case, Brownian rotation of the microspheres does not determine the characteristic frequency, since the nanoparticle dipoles relax by the Néel mechanism with characteristic frequencies beyond the experimental range. Complex magnetic susceptibility spectra of cobalt ferrite doped silica microspheres in dispersion are shown in figures 5 and 6 . When the cobalt ferrite nanoparticles are embedded in silica microparticles, rotational diffusion of the microspheres determines the characteristic frequency, $3.323 \mathrm{~Hz}$ for the microspheres in sample 1 and $0.416 \mathrm{~Hz}$ for the microspheres in sample 2. The curves through the measured points in figures 5 and 6 are least-squares fits of the spectra based on (7), (10a)-(10c) and a lognormal (1) distribution of the characteristic frequency $\omega_{\mathrm{B}}[16]$ around a value of $\left\langle\omega_{\mathrm{B}}\right\rangle$ with variance $\sigma^{2}$. From the characteristic frequencies, the hydrodynamic radii of the microparticles in sample 1 and sample 2 are obtained (see table 2). The low polydispersity in the hydrodynamic radii is in agreement with the observation of colloidal crystal formation in the particle sediments. The hydrodynamic radii from complex magnetic susceptibility measurements are comparable to the radius of single particles obtained from analysis of electron microscopy pictures (figure 2). The hydrodynamic radius is expected to be larger than the physical radius for several reasons. First, here a volumeaveraged radius is measured instead of the number-averaged sphere radius from image analysis of TEM pictures. Second, the hydrodynamic radius is calculated from the rotational diffusion coefficient (9) which increases as the particle volume fraction increases [25-27], which might lead to an overestimate of $R_{\mathrm{h}}$ as the sample is not infinitely diluted. Moreover, the irregular surface of the microspheres will contribute to a decrease in the rotational diffusion coefficient, resulting in a larger hydrodynamic radius. It should also be noted that the slightly porous silica microparticles tend to shrink under high vacuum in the electron microscope, which leads to an underestimate of the physical radius of the particles.

The zero-field magnetic moment of the microparticles can be calculated from the lowfrequency limit of the susceptibility spectrum using (8), and requires knowing the colloidal concentration $N_{\mathrm{mp}} / V$. In figures 5 and 6 , the microparticle concentration is $3.53 \times 10^{17} \mathrm{~m}^{-3}$ and $1.32 \times 10^{17} \mathrm{~m}^{-3}$ respectively. This corresponds to permanent magnetic dipole moments 

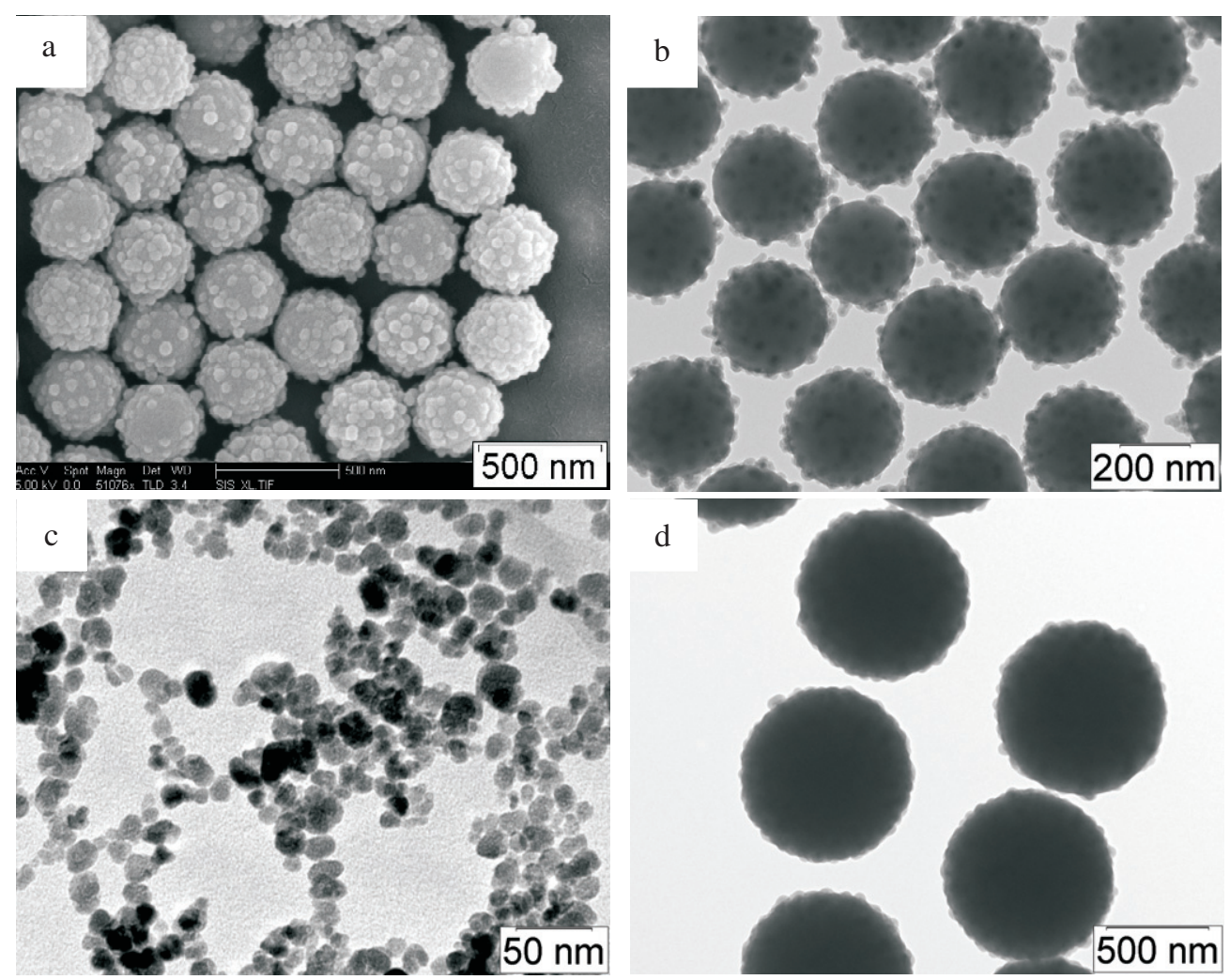

Figure 2. Electron microscopy pictures of the studied particles: (a) SEM picture and (b) TEM picture of $R \sim 200 \mathrm{~nm}$ silica-cobalt ferrite microspheres (sample 1), (c) TEM picture of the cobalt ferrite nanoparticles, and (d) TEM picture of $R \sim 400 \mathrm{~nm}$ silica-cobalt ferrite microspheres (sample 2). In the TEM pictures, the cobalt ferrite nanoparticles are visible as dark spots inside the silica matrix, and in the SEM picture, the silica-covered cobalt ferrite nanoparticles are seen as bright bulges on the surface of the silica core.

$\mu_{\mathrm{mp}}=3.03 \times 10^{-17} \mathrm{~A} \mathrm{~m}^{2}$ for the particles in sample 1 and $\mu_{\mathrm{mp}}=4.19 \times 10^{-17} \mathrm{~A} \mathrm{~m}^{2}$ for the particles in sample 2 . These values are higher than the magnetic moment of a single nanoparticle $\left(\mu_{\mathrm{np}}=1.61 \times 10^{-19} \mathrm{~A} \mathrm{~m}^{2}\right)$ but lower than the magnetic moment of a microparticle in a saturating magnetic field, as will be discussed later.

At $300 \mathrm{~Hz}$, the susceptibility is nearly zero, in line with the fact that all cobalt ferrite nanoparticles are embedded in microspheres (any free nanoparticles were removed during purification steps involving centrifugation) and that the dipole moments of most of the nanoparticles are blocked. In sample 1 , at $1 \mathrm{~Hz}$, Néel relaxation is responsible for only about $1.5 \%$ of the observed signal.

After treatment of sample 1 and sample 2 in a magnetic field of $2 \mathrm{~T}$, the magnetic silica particles have a larger magnetic dipole moment than prior to field exposure. This is revealed by the higher value of the low-frequency limit (figures 5 and 6). During the particle preparation, care had been taken not to make use of any magnet, to be able to compare the microparticle dipole moment before and after magnetization. In terms of the average magnetic moment of the nanoparticles the magnetic moment of the sample 1 particles increased from $188 \cdot \mu_{\mathrm{np}}$ to $260 \cdot \mu_{\mathrm{np}}$, an increase of $38 \%$. In sample 2 , the dipole moment increased from $355 \cdot \mu_{\mathrm{np}}$ to $520 \cdot \mu_{\mathrm{np}}$, an increase of $47 \%$. 

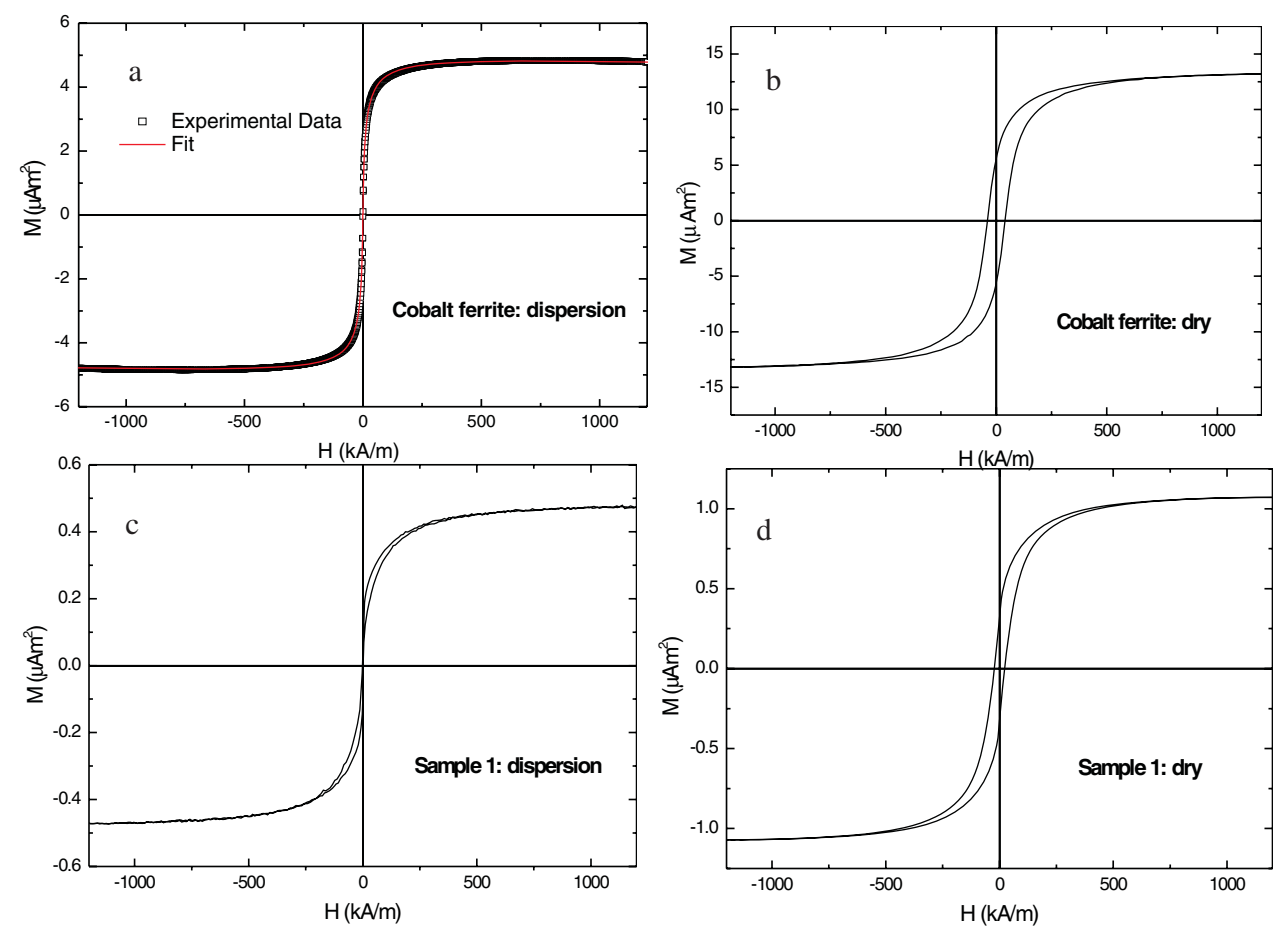

Figure 3. Magnetization curves of (a) cobalt ferrite nanoparticles in dispersion, (b) dry powder $(0.27 \mathrm{mg})$ of cobalt ferrite particles, (c) silica-cobalt ferrite microspheres (sample 1) in dispersion, and (d) dry powder $(0.52 \mathrm{mg})$ of sample 1 .

\subsection{Field-amplitude-dependent complex magnetic susceptibility}

The permanent magnetic moment of the silica particles was also determined from the fieldamplitude dependence of the low-frequency magnetic susceptibility. This method does not require a value of the concentration of particles in the dispersion to determine the permanent magnetic moment of the microspheres as with the previous method. In that case, the concentration might have been over- or underestimated due to an over- or underestimation of the density of the slightly porous silica particles. Measurements are presented in figure 7. As expected from (13), the susceptibility decreases when the magnetic field amplitude increases, which further confirms that the microparticles have a permanent magnetic moment in contrast to microparticles that only have an induced magnetic moment, which increases with the magnetic field, leading to an increase of the initial magnetic susceptibility [17, 18]. The cobalt ferrite doped magnetic silica particles can gain an induced magnetic moment as well, but to see the corresponding increase of the magnetic susceptibility, the magnetic field amplitude would have to be higher than here $\left(>1 \mathrm{kA} \mathrm{m}^{-1}\right)$. The magnetic moment of sample 1 before magnetic treatment determined from figure 7 is $\mu_{\mathrm{mp}}=3.08 \times 10^{-17} \mathrm{~A} \mathrm{~m}^{2}\left(\alpha / H=0.095 \mathrm{~m} \mathrm{~A}^{-1}\right)$. After magnetic treatment, the particles have gained a larger dipole moment, as seen also in the frequency-dependent susceptibility spectra. At low fields $\left(<300 \mathrm{~A} \mathrm{~m}^{-1}\right)$, the measured susceptibility decreases more quickly with the applied field with $\alpha / H=0.0131 \mathrm{~m} \mathrm{~A}^{-1}$, which corresponds to a dipole moment $\mu_{\mathrm{mp}}=4.25 \times 10^{-17} \mathrm{~A} \mathrm{~m}^{2}$. These values are in good agreement with the dipole moments obtained from the frequency-dependent spectra, which confirms that the number concentration in table 1 has been determined accurately. Surprisingly, 


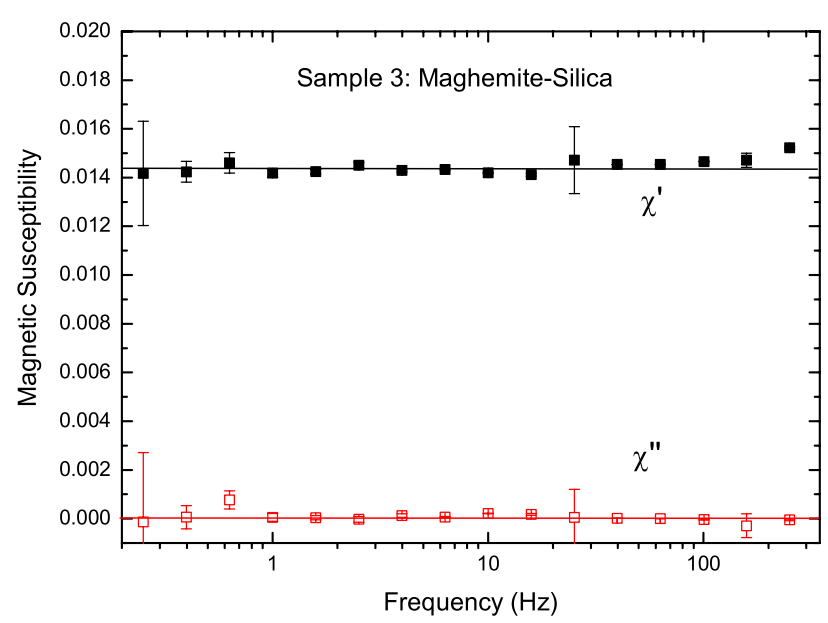

Figure 4. Low-field, low-frequency complex magnetic susceptibility spectrum of a dispersion of maghemite-doped silica microparticles (sample 3). The dipole moments inside the nanoparticles are not blocked, as would be the case for cobalt ferrite. Therefore the magnetic susceptibility is constant in the low-frequency range, and the Brownian rotation of such particles cannot be detected in the spectrum.

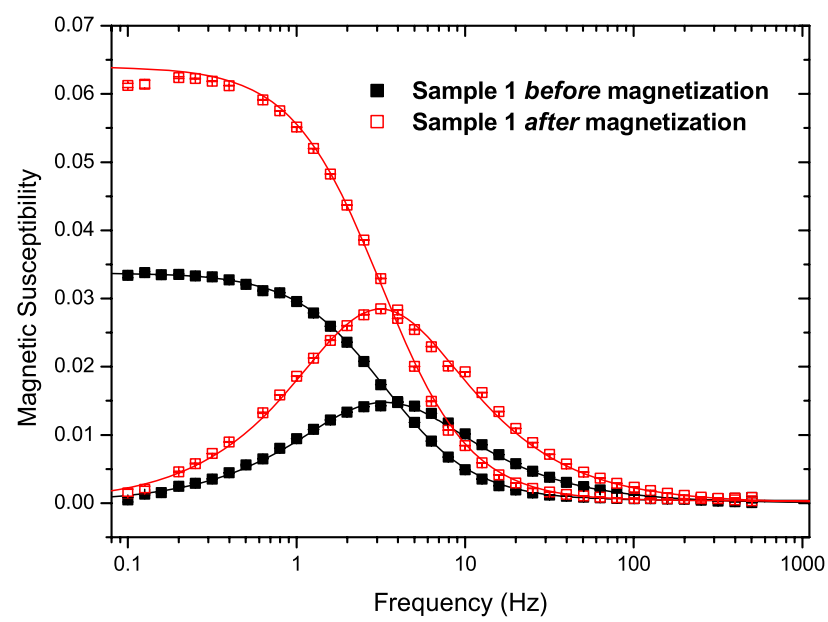

Figure 5. Complex magnetic susceptibility spectra of sample 1 before and after magnetic treatment. The value of $\chi_{\mathrm{LF}}$ increases by a factor 1.9 after magnetic treatment, which corresponds to an increase of the permanent microsphere dipole moment by $38 \%$. The theoretical fits are shown as full lines.

at higher fields ( $>300 \mathrm{~A} \mathrm{~m}^{-1}$ ), the amplitude dependence of the measured susceptibility for the magnetized sample is no longer described by (13). The only difference between the two measurements is the magnetic dipole moment of the particles; any chemical change of the sample on the timescale of the measurements is unlikely. Therefore, this effect at higher fields is likely to be related to the appearance of dipolar structures that break up as the field amplitude is decreased.

The results in figure 7 indicate that even when measuring at fields as low as $100 \mathrm{~A} \mathrm{~m}^{-1}$, there is a measurable deviation from the low-field approximation. A comparison of two 


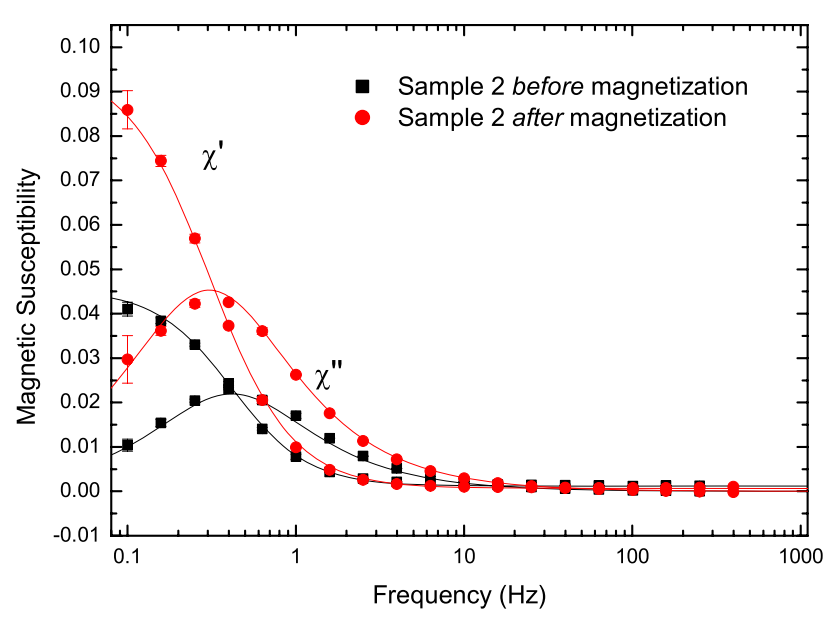

Figure 6. Complex magnetic susceptibility spectra of sample 2 before and after magnetic treatment. The value of $\chi_{\mathrm{LF}}$ increases by a factor 2.16 after magnetic treatment, which corresponds to an increase of the permanent microsphere dipole moment by $47 \%$. The theoretical fits are shown as full lines.

Table 2. Fit parameters and microsphere dipole moments obtained from the frequency spectra in figures 5 and 6 .

\begin{tabular}{lll}
\hline & Sample 1 & Sample 2 \\
\hline \multicolumn{3}{c}{ Before magnetization } \\
\hline$\omega_{\mathrm{B}} / 2 \pi(\mathrm{Hz})$ & $3.323 \pm 0.009$ & $0.416 \pm 0.005$ \\
$\sigma$ & $0.5400 \pm 0.0003$ & $0.12 \pm 0.01$ \\
$R_{\mathrm{h}}{ }^{\mathrm{a}}(\mathrm{nm})$ & $244 \pm 44$ & $487 \pm 20$ \\
$\chi_{\mathrm{LF}}+\chi_{\mathrm{HF}}$ & $0.03375 \pm 0.00003$ & $0.0453 \pm 0.0004$ \\
$\chi_{\mathrm{HF}}$ & $0.00038 \pm 0.00054$ & $0.0012 \pm 0.0005$ \\
$\mu_{\mathrm{mp}}(\mathrm{A} \mathrm{m})^{\mathrm{b}}$ & $3.03 \times 10^{-17}$ & $5.71 \times 10^{-17}$ \\
\hline \multicolumn{1}{c}{ After magnetization } \\
\hline$\omega_{\mathrm{B}} / 2 \pi(\mathrm{Hz})$ & $3.16 \pm 0.05$ & $0.307 \pm 0.002$ \\
$\sigma$ & $0.520 \pm 0.001$ & $0.333 \pm 0.011$ \\
$\chi_{\mathrm{LF}}+\chi_{\mathrm{HF}}$ & $0.0640 \pm 0.0004$ & $0.096 \pm 0.001$ \\
$\chi_{\mathrm{HF}}$ & $(0.000333 \pm 0.000096)$ & $(0.00067 \pm 0.00002)$ \\
$\mu_{\mathrm{mp}}(\mathrm{A} \mathrm{m})^{2}$ & $4.91 \times 10^{-17}$ & $8.37 \times 10^{-17}$ \\
\hline
\end{tabular}

a Obtained from (9) with $\eta=1.074 \mathrm{mPa}$ s and $T=295.5 \mathrm{~K}$.

b Obtained from (8) with the particle concentrations from table 1. The relative error of the dipole moment is not known, due to an unknown error in $N_{\mathrm{mp}} / V$.

frequency-dependent susceptibility spectra of sample 1 , measured at $H=57$ and $731 \mathrm{~A} \mathrm{~m}^{-1}$ respectively, is presented in figure 8 . In the low-frequency limit, for instance at $0.1 \mathrm{~Hz}$, the measured susceptibility decreases with the applied field amplitude as in figure 7. However, the characteristic frequency shifts from $\sim 4 \mathrm{~Hz}$ at $57 \mathrm{~A} \mathrm{~m}^{-1}$ to $\sim 7 \mathrm{~Hz}$ at $731 \mathrm{~A} \mathrm{~m}^{-1}$ and above $10 \mathrm{~Hz}$, the measurements at the two different amplitudes in figure 8 overlap. It is interesting to compare these results to the observations made by Fannin et al [18]. They observed an increase of the absorption peak frequency upon increasing the strength of a DC field superimposed on the AC field. In that case, the field-amplitude dependence was ascribed to a gradual size-dependent immobilization of polydisperse nanoparticles, where the largest 


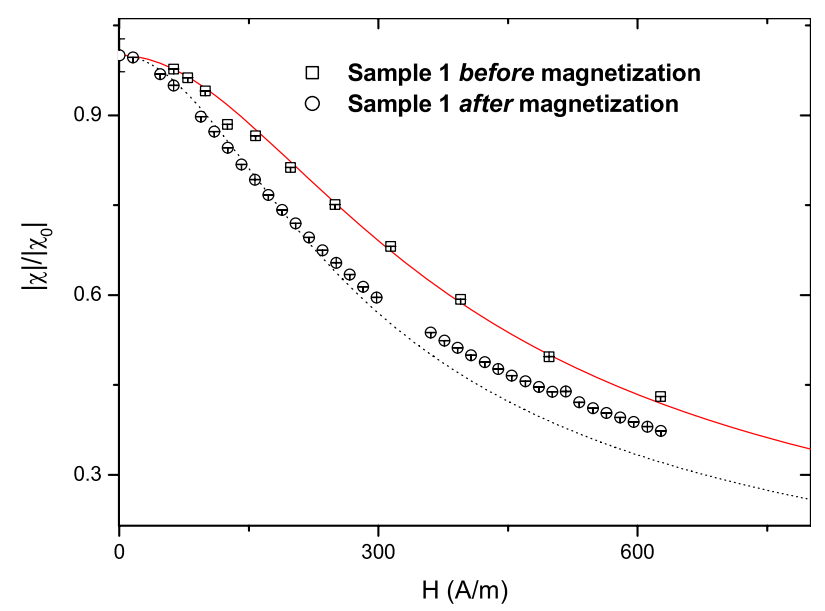

Figure 7. Measured susceptibility at $1 \mathrm{~Hz}$ of sample 1 before and after magnetic treatment as a function of the amplitude of the applied magnetic field. The solid line through the measured points is the expression in (13) with the fit parameter $\alpha / H=0.095 \mathrm{~m} \mathrm{~A}^{-1}$, which corresponds to a microsphere dipole moment, $\mu_{\mathrm{mp}}=3.08 \times 10^{-17} \mathrm{~A} \mathrm{~m}^{2}$, and the dotted line is (13) with the fit parameter $\alpha / H=0.0131 \mathrm{~m} \mathrm{~A}^{-1}$, which corresponds to a microsphere dipole moment $\mu_{\mathrm{mp}}=4.25 \times 10^{-17} \mathrm{~A} \mathrm{~m}^{2}$. The decrease in measured susceptibility as a function of field confirms that the microspheres have a permanent dipole moment. For the particles with a larger dipole moment, at fields higher than $300 \mathrm{~A} \mathrm{~m}^{-1}$ the data can no longer be described by (13).

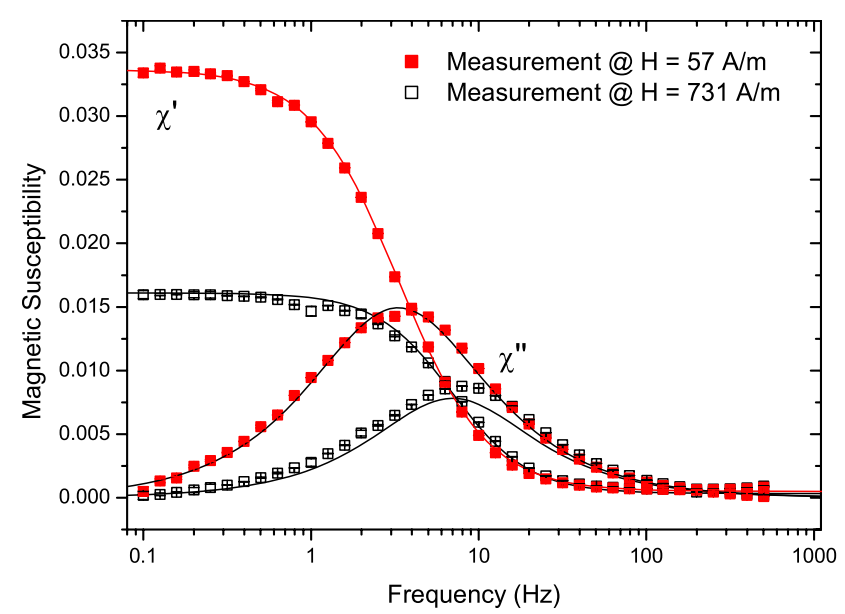

Figure 8. Susceptibility spectra of sample 1 measured at $H=57$ and $731 \mathrm{~A} \mathrm{~m}^{-1}$, respectively. In the latter case, the spectra are no longer measured in the low-field limit, and the measured susceptibility is therefore lower than at $57 \mathrm{~A} \mathrm{~m}^{-1}$, in agreement with figure 7 at low frequencies. At higher frequencies, the measured susceptibility is the same for both field amplitudes.

particles were slowed down first. In our case, the microspheres have a narrow size distribution; hence the same explanation cannot hold.

At low amplitude, the alternating field probes the rotational diffusion of the particles without having a major effect on the particle motion. However, at high amplitude, the magnetic torque on the particles is no longer negligible, but it speeds up their rotational motion, which leads to a shift of the absorption resonance to a higher frequency [19, 20]. Empirically, the 
amplitude dependence of the measured spectra can be fitted fairly well based on the following formulae:

$$
\begin{aligned}
& \chi^{\prime}=\chi_{0}^{\prime} F\left[H_{1} \frac{\omega_{0}^{2}}{\omega_{0}^{2}+\omega^{2}}\right] \\
& \chi^{\prime \prime}=\chi_{0}^{\prime \prime} F\left[H_{2} \frac{\omega_{0}^{2}}{\omega_{0}^{2}+\omega^{2}}\right]
\end{aligned}
$$

where $F(x)=L(x) /(x / 3)$ and $\chi_{0}^{\prime}, \chi_{0}^{\prime \prime}$, and $\omega_{0}$ are the low-amplitude values of $\chi^{\prime}, \chi^{\prime \prime}$, and $\omega_{\mathrm{B}}$. However, a good fit requires using field amplitudes $H_{1}$ and $H_{2}$ that differ from the experimental values $\left(H_{1}=550 \mathrm{~A} \mathrm{~m}^{-1}\right.$ and $H_{2}=1200 \mathrm{~A} \mathrm{~m}^{-1}$, whereas $\left.H_{\exp }=731 \mathrm{~A} \mathrm{~m}^{-1}\right)$. Moreover, at amplitudes above the weak-field limit, a full analysis should also include higher-order harmonic terms [19].

\section{Discussion}

The measurements clearly show that silica microparticles with embedded cobalt ferrite nanoparticles have a permanent magnetic dipole moment. The magnetic moment depends on the magnetic field-treatment history of the sample, which affects the orientation of the blocked nanoparticle dipoles. Before discussing the effect of magnetic treatment, we examine the theoretical case of randomly oriented dipoles. Finally, the interactions between the microspheres are discussed.

\subsection{Randomly oriented nanoparticle dipoles}

In zero field, the magnetization of a dispersion of particles with a permanent magnetic dipole moment is zero. For every dipole oriented one way, another dipole is present that is oriented in the opposite direction, so that oppositely oriented dipoles cancel each other's contributions to the total magnetic moment. However, when magnetic nanoparticles are embedded in a single microsphere, the number of embedded nanoparticle dipoles is limited and they do not cancel statistically.

To calculate the magnetic moment of a microparticle, the vector sum of the individual dipoles must be determined. The vector sum of $N$ randomly oriented unit vectors is on average equal to $\sqrt{N}$. Therefore, if all individual dipoles of the microparticles are randomly oriented, the average dipole moment of one microparticle would be $\sqrt{N} \mu_{1}$, where $\mu_{1}$ is the magnetic dipole moment of a nanoparticle. Random orientation means that all orientations are equally probable, but it does not mean that exactly the same number of dipoles is oriented in opposite directions. A direct analogue is the effect of a random walk: displacement is equally probable in all directions, but nevertheless the average displacement from the starting point is not zero but scales with the square root of the number of steps.

The results from our measurements on sample 1 show that the average number of nanoparticles per microparticle is approximately $N=900$. The corresponding microsphere dipole moment in zero field is expected to be of the order of $\sqrt{900} \cdot \mu_{1}=30 \cdot \mu_{1}$ if all the nanoparticle dipoles are randomly oriented, assuming that all the nanoparticles have blocked dipole moments. The actual magnetic moment of the as-synthesized microspheres was found to be about $190 \cdot \mu_{1}$, more than six times the random-orientation value. During the synthesis, care was taken not to expose the particles to an external magnetic field other than the earth's magnetic field, which is approximately $40 \mathrm{~A} \mathrm{~m}^{-1}$ and has a negligible interaction $\mu_{0} \mu \mathrm{H}$ of about $2 \times 10^{-3} k_{\mathrm{B}} T$ with the nanoparticles. One possible explanation for the magnetic moment of the microparticles being larger than the random-orientation value is interaction between the 


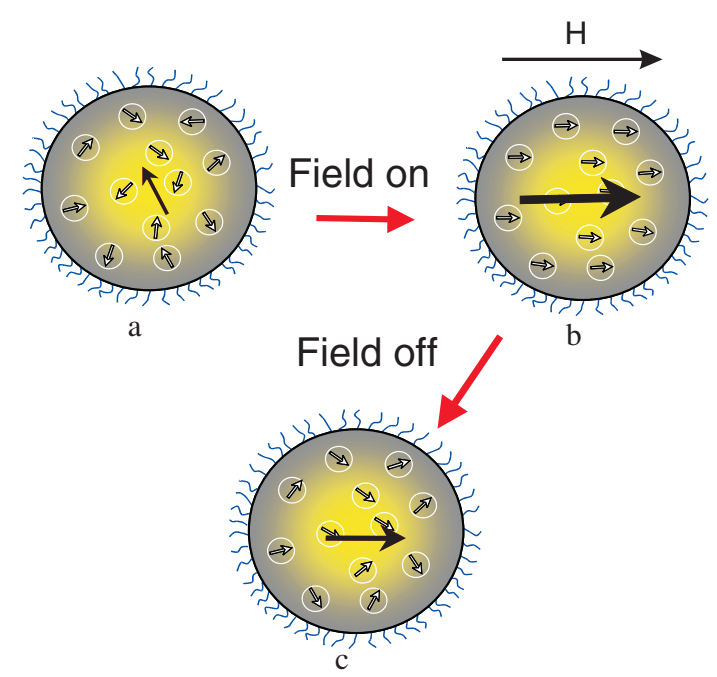

Figure 9. Schematic illustration showing silica microspheres with embedded cobalt ferrite nanoparticles: (a) as synthesized, exposed only to the earth magnetic field, (b) while exposed to a strong external magnetic field, causing all individual dipole moments of the nanoparticles to align in the field direction, resulting in a large induced microsphere dipole moment, and (c) after removing the external magnetic field, upon which the nanoparticle dipole moments relax back to the easy axis closest to the direction of the applied magnetic field, resulting in a microsphere dipole moment which is smaller than in (b) but larger than in (a).

nanoparticle dipoles. The dipole-dipole interaction between two $d=10 \mathrm{~nm}$ cobalt ferrite particles in head-to-tail configuration at contact is about $1.3 k_{\mathrm{B}} T$, calculated using

$$
V=-\frac{\mu_{0} \mu_{1}^{2}}{2 \pi d^{3}}
$$

where $d$ is the diameter of the core of a nanoparticle. This interaction is sufficiently strong to block the dipole orientations if each nanoparticle interacts with more than one neighbour. Moreover, larger particles in the size distribution will experience much stronger dipole-dipole interactions than the average value of $1.3 k_{\mathrm{B}} T$. Strong dipolar interactions between the larger nanoparticles can be present already during the synthesis of the microspheres, which might lead to nanoparticles forming aggregates before they attach to the silica surface. Consequently, within these aggregates, the nanoparticle dipole moments will not have a random orientation. It can be concluded that significant dipolar coupling occurs between the nanoparticles in the microspheres.

\subsection{Effect of magnetic field treatment}

When a magnetic field is applied, the microparticles gain an induced magnetic dipole moment, and when the magnetic field is sufficiently strong, the zero-field magnetic dipole moment is changed as illustrated in figure 9. Before magnetic treatment, the as-synthesized particles have a permanent magnetic moment as discussed in section 5.1. During treatment in a strong magnetic field, for instance at $2 \mathrm{~T}$, all the nanoparticle dipoles are aligned. Once the field is removed, the dipoles which are blocked in zero field relax to an easy axis of magnetization. Before such magnetic treatment, the vector components of the dipoles along the field direction can have random signs, but after magnetic treatment, that vector component is in the direction of the field instead of the opposite direction, at least in the limit case. This is because when the 
strong magnetic field is removed, the dipoles are expected to relax to the easy-axis direction that is closest to the field direction. The expected dipole moment would now be $2 N \mu_{1} / 3$ : the vector component along the field direction scales with $\cos (\theta)$, where $\theta$ is the angle with the field direction, and the integral of $\cos (\theta)$ from 0 to $\pi$ is $2 / 3$.

From the low-field limit of the complex magnetic susceptibility spectrum of sample 1 , the calculated dipole moment of the magnetically treated particles is $260 \cdot \mu_{1}$ instead of the expected value of $2 \cdot 900 \cdot \mu_{1} / 3=600 \cdot \mu_{1}$. Even though the magnetic moment of the microparticles has increased, the limiting case is not reached. Moreover, in the magnetization curves of dry sample 1 (figure 3) the remanence $\left(M_{\mathrm{r}} / M_{\mathrm{s}}\right)$ is not $2 / 3=0.667$ but 0.305 for the sample 1 particles. This is lower than 0.425 for the dry nanoparticles that are not embedded in microparticles. The remanence is higher for the nanoparticles than for the microparticles, probably because of dipole-dipole interactions. The dry nanoparticles are present in a compact 3D network, while on the silica particles the cobalt ferrite particles are spread over a (2D) surface as observed with electron microscopy.

\subsection{Dipolar and hydrodynamic interactions}

From the permanent dipole moments of the magnetic silica particles, the dipole-dipole interactions between the particles can be estimated. The maximum magnetic interaction between two composite particles using (18) with $d=2 R_{\mathrm{h}}=468 \mathrm{~nm}$ is about $0.4 k_{\mathrm{B}} T$ for the microparticles in sample 1 and $0.8 k_{\mathrm{B}} T$ for magnetically treated sample 1 . For doublets or larger dipolar structures in zero field, the head-to-tail dipolar pair interaction energy should exceed $2 k_{\mathrm{B}} T$ [21-23]. Clearly, no dipolar structures are expected in the magnetic silica dispersions. Their absence is confirmed by the complex susceptibility spectra, in which dipolar structures would not just show the Brownian rotation of single microparticles but also that of dipolar structures, with several times lower characteristic frequencies for Brownian rotation than for single microspheres [7]. Moreover, the susceptibility would not become constant just below the characteristic frequency for single microparticle rotation. It is noted that equation (18) is not strictly applicable to the microparticles because they are not point dipoles. It only gives an estimate of the interaction between composite microparticles. The magnetic material is in a shell near the surface of the spheres. The dipolar interaction between two magnetic microparticles in this case is therefore stronger than it would have been if all the nanoparticles were embedded at the centre of the microparticles. Although insufficient for aggregation, interactions of $1 k_{\mathrm{B}} T$ or less between microparticles might still affect the Brownian rotation in a detectable way. In the complex magnetic susceptibility spectra of samples 1 and 2 (see figures 5 and 6), the characteristic frequencies are slightly shifted to lower frequencies after increasing the dipole moment by treatment in a strong magnetic field. Since the concentration of particles was constant, this indicates a decreasing rotation rate due to dipolar interactions. In other experiments, the same decrease in the rotation rate could be due to adsorption of molecules at the surface of the microparticles, which increases the hydrodynamic radius of the particles. The latter effect can be used to develop highly sensitive biomolecular sensing procedures, as was demonstrated by Astalan et al [24]. The interaction of the microspheres with even larger objects could drastically slow down the motion of the microspheres. We are currently investigating the rotational dynamics of our microspheres with a permanent magnetic moment at various concentrations including confined geometries such as colloidal crystals and glasses.

\section{Conclusions}

Magnetic silica microparticles with embedded cobalt ferrite nanoparticles have a permanent magnetic moment. This is revealed by a relaxation in the complex magnetic susceptibility 
spectrum at the frequency corresponding to Brownian rotation of the microparticles. The magnetic moment of the magnetic composite particles can be calculated from the initial magnetic susceptibility, if the concentration of the dispersion is known. Alternatively, the initial susceptibility can be measured as a function of the magnetic field amplitude. The susceptibility decreases with increasing magnetic field amplitude, due to the non-linearity of the Langevin equation, and from that the microparticle dipole moment can be calculated without knowing the particle concentration. The magnetic moment of the microparticles before and after magnetization in a strong magnetic field can be understood in terms of the orientations of the embedded nanoparticle dipoles, along the easy axis of magnetization in the absence of an external field. Even if the dipoles had random orientations, the vector sum of the nanoparticle dipoles of each microparticle would be non-zero and, in our case, dipolar coupling between the nanoparticles also affects the orientation of the as-synthesized particles before exposure to a strong magnetic field. The permanent magnetic moment of the microparticles increases further after exposure to a strong magnetic field, since the dipole orientations do not relax to a random distribution when the field is removed.

\section{Acknowledgments}

We thank Stefano Sacanna for taking the SEM pictures. This work was financially supported by OCÉ Technologies, Venlo, The Netherlands.

\section{References}

[1] Pankhurst Q A, Connolly J, Jones S K and Dobson J 2003 J. Phys. D: Appl. Phys. 36 R167

[2] Claesson E M and Philipse A P 2005 Langmuir 219412

[3] Piazza R and Degiorgio V 1991 Phys. Rev. Lett. 673868

[4] Koenderink G H, Lettinga M P and Philipse A P 2002 J. Chem. Phys. 1177751

[5] Lettinga M P, Koenderink G H, Kuipers B W M, Bessels E and Philipse A P 2004 J. Chem. Phys. 1204517

[6] Berkovski B and Bashtovoy V (ed) 1996 Magnetic Fluids and Applications Handbook (New York: Begell House) p 7

[7] Rosensweig R E 2002 J. Magn. Magn. Mater. 252370

[8] Erné B H, Claesson E M, Sacanna S, Klokkenburg M, Bakelaar I A and Kuipers B W M 2006 J. Magn. Magn. Mater. doi:10.1016/j.jmmm.2006.11.169

[9] Lide D R (ed) 2000 Handbook of Chemistry and Physics (Boca Raton, FL: CRC Press) pp 6-246

[10] Erné B H, Butter K, Kuipers B W M and Vroege G J 2003 Langmuir 198218

[11] Fannin P C 1998 Adv. Chem. Phys. 104181

[12] Tourinho F A, Franck R and Massart R 1990 J. Mater. Sci. 253249

[13] Massart R, Dubois E, Cabuil V and Hasmonay E 1995 J. Magn. Magn. Mater. 1491

[14] Philipse A P and Vrij A 1989 J. Colloid Interface Sci. 128121

[15] Kuipers B W M, Bakelaar I A, Klokkenburg M and Erné B H 2006 in preparation

[16] Erné B H, vander Pol E, Vroege G J, Visser T and Wensink H H 2005 Langmuir 211802

[17] Fannin P C, Cohen-Tannoudji L, Bertrand E, Giannitsis A T, Oireachtaigh C M and Bibette J 2006 J. Magn. Magn. Mater. 303147

[18] Fannin P C, Charles S W, Oireachtaigh C M and Odenbach S 2006 J. Magn. Magn. Mater. 3021

[19] Felderhof B U and Jones R B 2003 J. Phys.: Condens. Matter 15 S1363

[20] Caizer C 2005 J. Phys.: Condens. Matter 172019

[21] Butter K, Bomans P H, Frederik P M, Vroege G J and Philipse A P 2003 Nat. Mater. 288

[22] Klokkenburg M, Vonk C, Claesson E M, Meeldijk J D, Erné B H and Philipse A P 2004 J. Am. Chem. Soc. 12616706

[23] Klokkenburg M, Dullens R P A, Kegel W K, Erné B H and Philipse A P 2006 Phys. Rev. Lett. 960372031

[24] Astalan A P, Ahrentorp F, Johansson C, Larsson K and Krozer A 2004 Biosens. Bioelectron. 19945

[25] Degiorgio V, Piazza R and Jones R B 1995 Phys. Rev. E 522707

[26] Watzlawek M and Nägele G 1997 Prog. Colloid Polym. Sci. 104168

[27] Hagen M H J, Frenkel D and Lowe C P 1999 Physica A 272376 